\title{
Embryonic vascular endothelial cells are malleable to reprogramming via Prox1 to a lymphatic gene signature
}

\author{
Harold Kim¹, Vicky PKH Nguyen'1, Tatiana V Petrova2,3, Maribelle Cruz', Kari Alitalo² and Daniel J Dumont*1
}

\begin{abstract}
Background: In vivo studies demonstrate that the Prox 1 transcription factor plays a critical role in the development of the early lymphatic system. Upon Prox 1 expression, early lymphatic endothelial cells differentiate from the cardinal vein and begin to express lymphatic markers such as VEGFR-3, LYVE-1 and Podoplanin. Subsequent in vitro studies have found that differentiated vascular endothelial cells can be reprogrammed by Prox 1 to express a lymphatic gene profile, suggesting that Prox 1 can initiate the expression of a unique gene signature during lymphangiogenesis. While the in vitro data suggest that gene reprogramming occurs upon Prox 1 expression, it is not clear if this is a direct result of Prox 1 in vascular endothelial cells in vivo.
\end{abstract}

Results: Overexpression of Prox 1 in vascular endothelial cells during embryonic development results in the reprogramming of genes to that of a more lymphatic signature. Consequent to this overexpression, embryos suffer from gross edema that results in embryonic lethality at E13.5. Furthermore, hemorrhaging and anemia is apparent along with clear defects in lymph sac development. Alterations in junctional proteins resulting in an increase in vascular permeability upon Prox 1 overexpression may contribute to the complications found during embryonic development.

Conclusion: We present a novel mouse model that addresses the importance of Prox 1 in early embryonic lymphangiogenesis. It is clear that there needs to be a measured pattern of expression of Prox 1 during embryonic development. Furthermore, Prox 1 reprograms vascular endothelial cells in vivo by creating a molecular signature to that of a lymphatic endothelial cell.

\section{Background}

The specialization of the vasculature is driven by a number of molecular pathways that dictate the fate of blood vessels to that of an artery or vein [1]. Following this initial programming, the development of the lymphatic system is dependent on the further differentiation of the venous endothelium. The newly differentiated lymphatic endothelial cells migrate in a polarized fashion to coalesce into an early lymph sac, which then progress to form the lymphatic vasculature proper $[2,3]$.

A number of genes have been found to be associated with the lymphatic endothelial cell profile, for example LYVE-1, Podoplanin and VEGFR-3 [4-6]. In addition to

\footnotetext{
*Correspondence: dan.dumont@sri.utoronto.ca

1 Sunnybrook Research Institute University of Toronto 2075 Bayview Avenue Toronto, Ontario, M4N 3M5, Canada

Full list of author information is available at the end of the article
}

this profile, the transcription factor Prox1 has been found to act as an important regulatory switch, altering the molecular identity of venous endothelium and imparting them with attributes that augment lymphatic development. Indeed, genetic ablation of prox 1 results in embryonic lethality; hemizygous null mice are found to die shortly after birth [3]. In both cases, a major developmental defect was found to involve the emerging lymphatic system. Specifically, the early lymphatic endothelial cells (LECs) that normally bud off from the cardinal vein (CV) in a polarized manner do not do so. Instead, prox 1 null LECs do not migrate, resulting in defective lymph sac formation and consequently no development of a functional lymphatic system. Furthermore, these null cells molecularly default to a more vascular-like phenotype. To this end, prox 1 null mice display lymphedema and chylous ascites buildup resulting in death $[2,3]$. 
It is clear that vascular endothelial cells require the regulated expression of Prox1 early in development, initiating a transcriptional program that results in lymphatic differentiation. This point underscores the plasticity of differentiated endothelial cells under the influence of Prox1. Indeed, microarray analysis of vascular endothelial cells engineered to overexpress Prox1 resulted in the upregulation of lymphatic specific genes while downregulating a number of blood specific genes $[7,8]$; this study extending the known surface markers of lymphatic endothelial cells by providing a more in-depth characterization of LEC gene signature. The in vivo knockout data demonstrates that prox 1 plays an important role in early lymphatic development. Furthermore, targeted deletion of prox 1 within vascular endothelial cells recapitulates the lymphatic defect and the edematous phenotype [9]. Moreover, in vitro studies suggest that this lymphatic defect is potentially due to an inability to molecularly reprogram early vascular endothelial cells to a lymphatic fate $[7,8]$. Given this however, it is not clear whether the reprogramming to a specific gene signature does indeed occur in vivo. Here we describe an in vivo model to address the molecular significance of Prox1 in reprogramming vascular endothelial cells to that of a lymphatic gene profile.

\section{Results}

\section{Prox 1 expression in double transgenic mice}

In order to investigate whether Prox1 is necessary and sufficient in regulating cell fate, we have generated a mouse model that forces the expression of Prox 1 specifically in vascular endothelial cells. Our transgenic model takes advantage of a bigenic expression system driven by the tie 1 promoter (Figure 1a). This model results in targeted expression within vascular compartments such as the cardinal vein as well as the dorsal aorta (Figure $1 \mathrm{~b}$ and 1c, see Additional file 1). While the expression of Prox1 in DT embryos is also found on both arteries and veins, both these vascular structures appear to differentially overexpress Prox-1 throughout development in our model suggesting that arterial and venous endothelial cells may regulate Prox-1 RNA/protein levels in a different fashion. While the directionality of the budding lymphatic endothelial cells appear to be more tightly regulated in control embryos, DT embryos appear to display more Prox 1 positive cells in the periphery. These results suggest that normally, Prox1 expression and lymphatic endothelial cell budding are tightly controlled and can be disrupted by the overexpression of Prox 1 in the developing vasculature.

\section{Phenotype of DT Prox 1 mice}

As development progresses, both the vascular and lymphatic system matures to form two separate yet critically

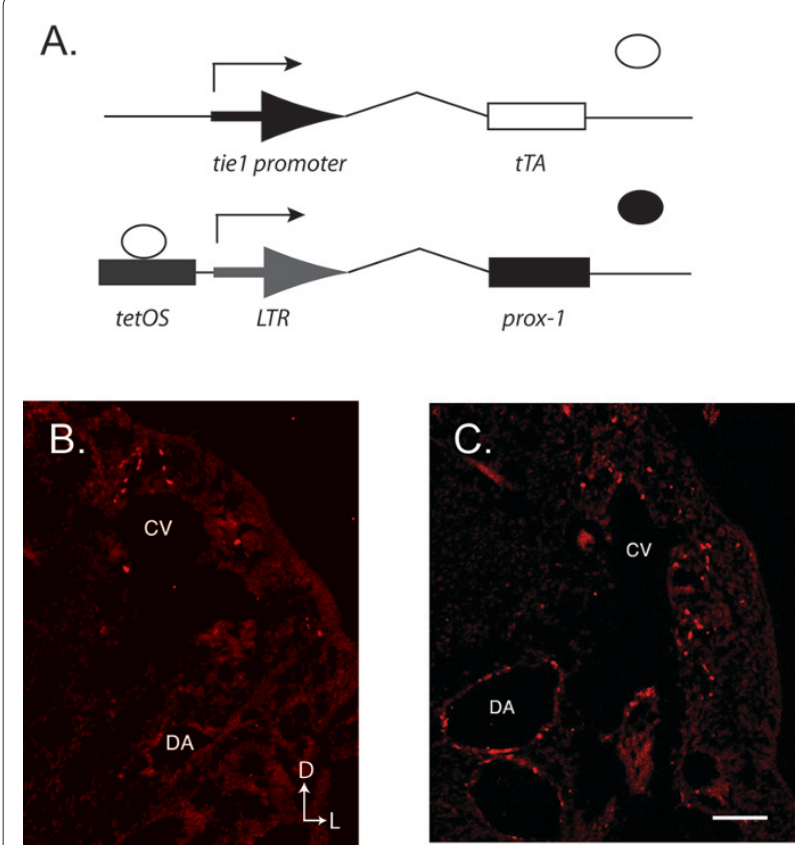

Figure 1 Bigenic model for Prox1 overexpression. (A) The prox 1:tetOS transgene is driven by the tissue specific tie 1:tTA promoter. Open circle: tTA, closed circle: Prox 1 protein. (B) The expression of Prox 1 in E10.5 embryos displays a polarized expression pattern specifically from the cardinal vein. (C) In contrast, overexpression of Prox 1 in the double transgenic (DT) embryos display an increase in Prox 1 positive cells from the cardinal vein. Significantly, Prox 1 is also present on the dorsal aorta, which is normally negative for Prox 1 expression. Orientation arrows for B and C: D- Dorsal, L- Lateral. Scale bar: $50 \mu \mathrm{m}$.

interrelated circuits. In early development, there are no overt deficiencies in the blood vasculature of DT embryos. However, by E10.5 we begin to observe small hemorrhages on the surface of developing DT embryos (Figure 2a and 2b, arrorwheads). At later stages in development we notice some subtle delays in growth, more profound blood loss, with blood pooling within the embryo (Figure $2 \mathrm{c}$ and $2 \mathrm{~d}$ ). In the most severe embryonic cases we find anemic embryos that display massive edema at E13.5 where the epidermis separates from the body as a result of trapped extravasated fluid (Figure 2e and $2 \mathrm{f}$, double arrowhead). Upon further analysis, DT embryos that harbor the most severe phenotypes also display enlarged lymph sacs relative to their control counterparts (Figure 2e and 2f). These results suggest that the overexpression of Prox 1 can pose a developmental crisis during early gestation. Indeed, 33\% of postnatal DT's do not make the expected Mendelian double transgenic ratio (Table 1). However, the majority of the postnatal DT's do appear to survive to birth. These results suggest that the overexpression of Prox 1 can have deleterious consequences in the developing embryo correlating with hemorrhaging, anemia and edema. 
Table 1: Analysis of number of embryos and postnatal births.

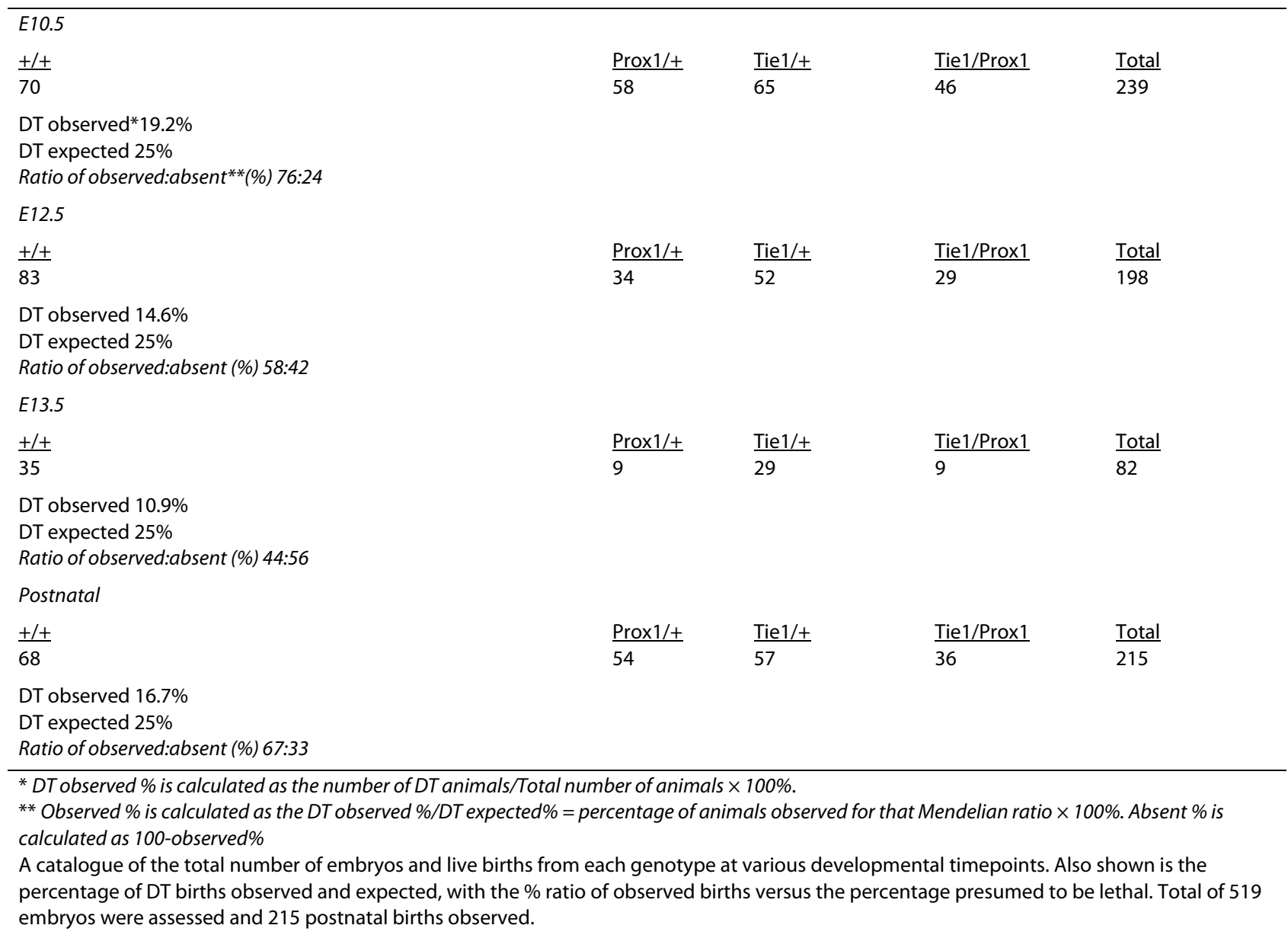

\section{Marker analysis of yolk sacs from Prox1 DT mice}

Given the influence of Prox1 on gene regulation in vascular and lymphatic endothelial cells, we further investigated the possibility of a switch in the molecular identity of endothelial cells using markers that are influenced by Prox1. To this end, we started our analysis with the yolk sac, a relatively simple tissue that is rich in blood vasculature (Figure 3a). Analysis of control or DT embryos reveal that the overexpression of Prox1 in vascular endothelial cells could alter gene expression to that of a more lymphatic endothelial cell signature. For example, transcripts for genes such as VEGFR-3 and CyclinE2 increase while VEGFR-2, Neuropilin1 and Stat6 decrease (Figure 3b). Furthermore, protein levels from yolk sacs derived from DT embryos demonstrate that VEGFR-2 and Tie2 decrease while Neuropilin-2 increases with a marginal increase in VEGFR-3. Wholemount analysis of DT yolk sacs support the reprogramming event, indicated with an increase in Podoplanin expression that correlates with Prox1 overexpression (data not shown). Consistent with previous findings, the overexpression of Prox1 in vascular endothelial cells appear to sufficiently alter the gene signature of BECs to that of a more LEC profile in vivo.

\section{Jugular veins from Prox1 DT embryos display lymphatic markers}

To visualize the potential of Prox1 in reprogramming the vascular endothelium, markers such as VEGFR-3 and Podoplanin were used to analyze the developing jugular vein (JV). Within the DT samples, it was found that the levels of VEGFR-3 increased relative to that observed in the control embryos (Figure 4a versus b, arrows). Previous studies by Schacht et al. show that Podoplanin expression is tightly regulated during development, being expressed early on the JV and gradually being downregulated by E13.5. This downregulation coincides with a shift in expression to the developing lymph sacs derived from the same JV [5]. Interestingly, at the timepoint where Podoplanin expression should be downregulated on the jugular vein, DT embryos show strong expression of Podoplanin relative to controls (Figure 4c versus d, arrows). These results further suggest that the overexpression of Prox1 can alter the expression pattern of vas- 
cular endothelial cells to that of a more lymphatic signature.

\section{Overexpression of Prox 1 correlates with changes in junctional proteins in vivo and in vitro}

With the overexpression of Prox1, changes in gene signatures are observed in the developing embryo. These changes correlate with defects in embryonic development such as edema and hemorrhaging. While it suggests that the overexpression of Prox 1 in the early developing vasculature is causal to these defects, the functional explanation for the edema and hemorrhaging is unclear. One possibility that may explain the observed developmental defects may lie in changes in vessel permeability upon Proxl overexpression. To investigate this possibility, proteins that are associated with tight junctions were analyzed from yolk sacs of control and DT embryos. Interestingly, the overexpression of Prox 1 results in the alteration of junctional proteins from the normal pattern of expression such as ZO-1, Occludin and to a lesser extent JAM-1 (Figure 5a). Further analysis using cultured venous endothelial cells [10] show that overexpression of Prox1 results in a similar trend of misregulated junctional targets such as ZO-1 and PECAM-1 (Figure 5b), these changes correlating with an altered cellular morphology (Figure 5c). To further define the consequences of Prox1 overexpression in VECs, an in vitro permeability assay via Boyden chamber was performed comparing control VECs with those that overexpress Prox1. Significantly, VECs that overexpress Prox1 display a higher degree of permeability, assessed by the passage of FITC-albumin, when compared to control VECs (Figure 5d). This suggests that VECs become more permeable with Prox1 overexpression, potentially due to alterations in the integrity of cell-cell junctions.

\section{Discussion}

The importance of Prox1 in initializing differentiation was shown to be a result of its ability to reprogram blood endothelial cells to a lymphatic endothelial cell profile. This molecular switch shown in vitro suggested that Prox1 initiates the transcriptional machinery necessary for lymphangiogenesis $[8,11]$. While the Prox1 knockout study as well as a tissue specific deletion of Prox1 in the endothelium [9] point to the necessity of Prox1 in lymphatic development, it does not provide a complete extension on the molecular players that confer the ability to reprogram the blood vasculature as suggested from in vitro studies $[7,8]$. To this end, one way to address this involves the specific overexpression of Prox 1 in the endothelial cell compartment. In the present study, we examine the importance of vascular reprogramming and fate specificity in vivo with a tie1/proxl bigenic mouse model that specifically expresses Prox1 in early vascular endothelial cells.

Early expression of Prox1 in vascular endothelial cells result in no overt embryonic phenotype, however as development progresses Prox1 double transgenic embryos appear growth delayed and display more overt bleeding. Of the most serious cases, DT embryos are anemic and edematous. This correlated with the alteration of a number of molecular targets associated with vascular reprogramming such as an increase in VEGFR-3, Neuropilin-2 and STAT6 and a decrease in Neuropilin-1, VEGFR-2, Tie-2 and CyclinE2 $[7,8,12]$. Examination of the most severely affected DT embryos show a general degradation of the embryonic structure, separation of the epidermis and enlarged lymph sacs when compared to their wild type counterparts. Furthermore, vascular structures such as the jugular vein, which normally express low levels of VEGFR-3 and Podoplanin are now highly positive for these lymphatic markers. In conjunction with previous data demonstrating the importance of Prox1 in BEC differentiation to LECs, we present data that provides further in vivo evidence of the molecular players involved in the reprogramming of BECs to LECs early in lymphatic vascular development. Consistent with our data, it was recently observed that Sox 18 is upstream of Prox1; in vitro overexpression of Sox18 in embryonic stem cells and vascular endothelial cells results in the upregulation of Prox 1 and an increase in lymphatic markers such as Podoplanin, indirectly confirming our data presented here [13]. In addition, the conditional deletion of Prox1 results in the dedifferentiation of lymphatic endothelial cells to that of a more vascular endothelial cell-like phenotype [14]. This further suggests that Prox1 is critical for the maintenance of the lymphatic endothelial cell fate, and that endothelial cells are genetically malleable. Our in vivo study further confirms the importance of Prox1 in generating a lymphatic profile by altering the gene signature of vascular endothelial cells.

One consequence that results from the overexpression of Prox1 in the vascular compartment of the developing embryo is edema. At the histological level, we observe the separation of the dermal layer from the epidermis as a result of fluid leakage, characteristic of an improper lymphatic drainage system. Furthermore, a prominent feature of the most severely affected embryos is the enlargement of the lymph sacs. It is unclear as to the mechanism of the increased size, whether it is from an increase in the number of differentiating early lymphatic endothelial cells budding from the jugular vein, or from an increase in proliferation of committed lymphatic endothelial cells that are in the process of or have already formed the lymph sac. Interestingly, early analysis of E10.5 DT embryos show an increase in Prox1 positive 


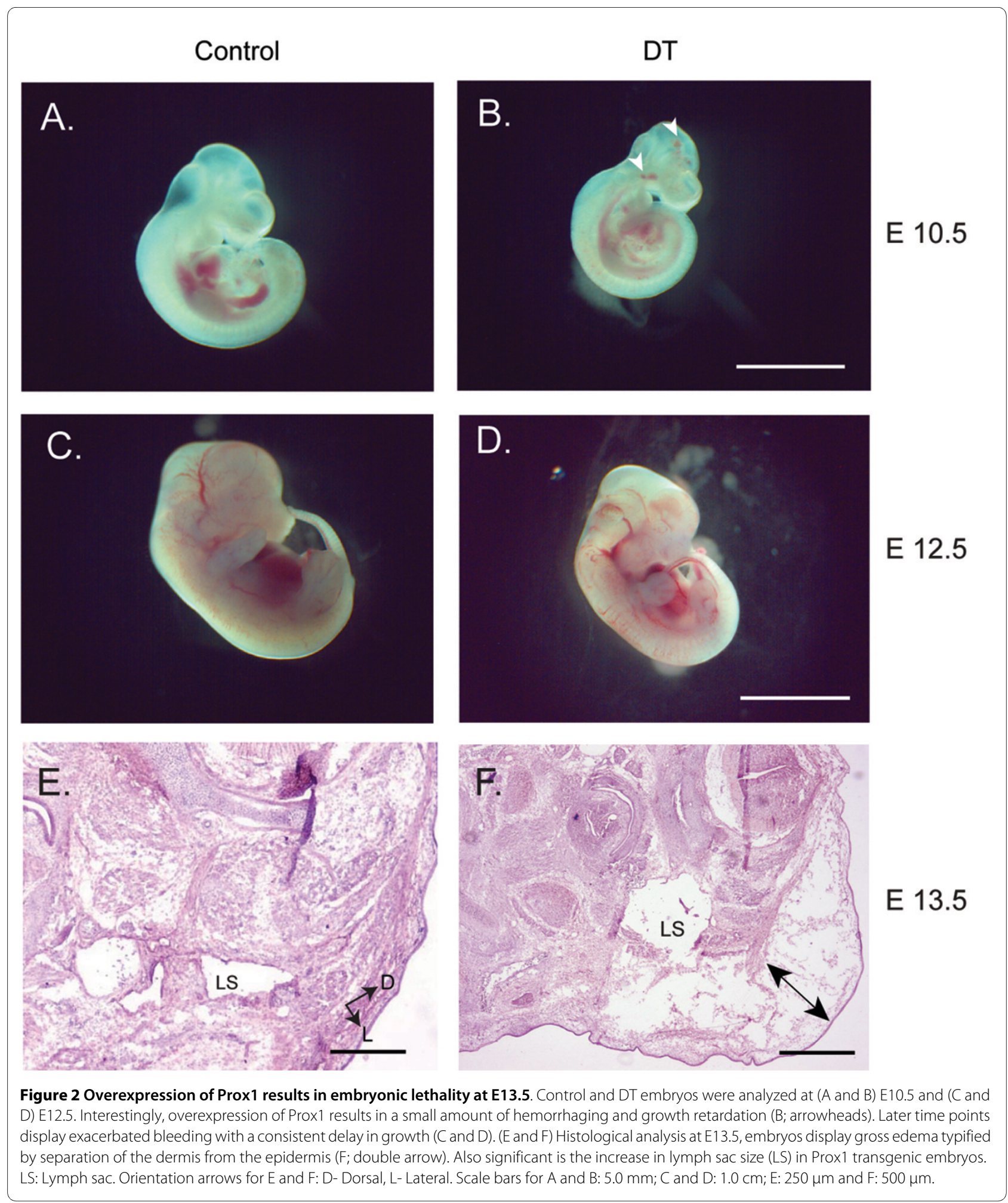

cells in the periphery; this increase in population may play a contributing factor to the increase in lymph sac size. The potential for an increase in proliferation due to Prox1 overexpression is consistent with previously published work that identifies the upregulation of a number of cell cycle targets such as CyclinE2 and p57kip [7].
The data suggests that the overexpression of Prox 1 during early embryogenesis results in the reprogramming of vascular endothelial cells to a more lymphatic cell fate resulting in edema, hemorrhaging and death. While it demonstrates the importance of tightly regulating Prox1 in development, it is unclear as to why the hemorrhaging 


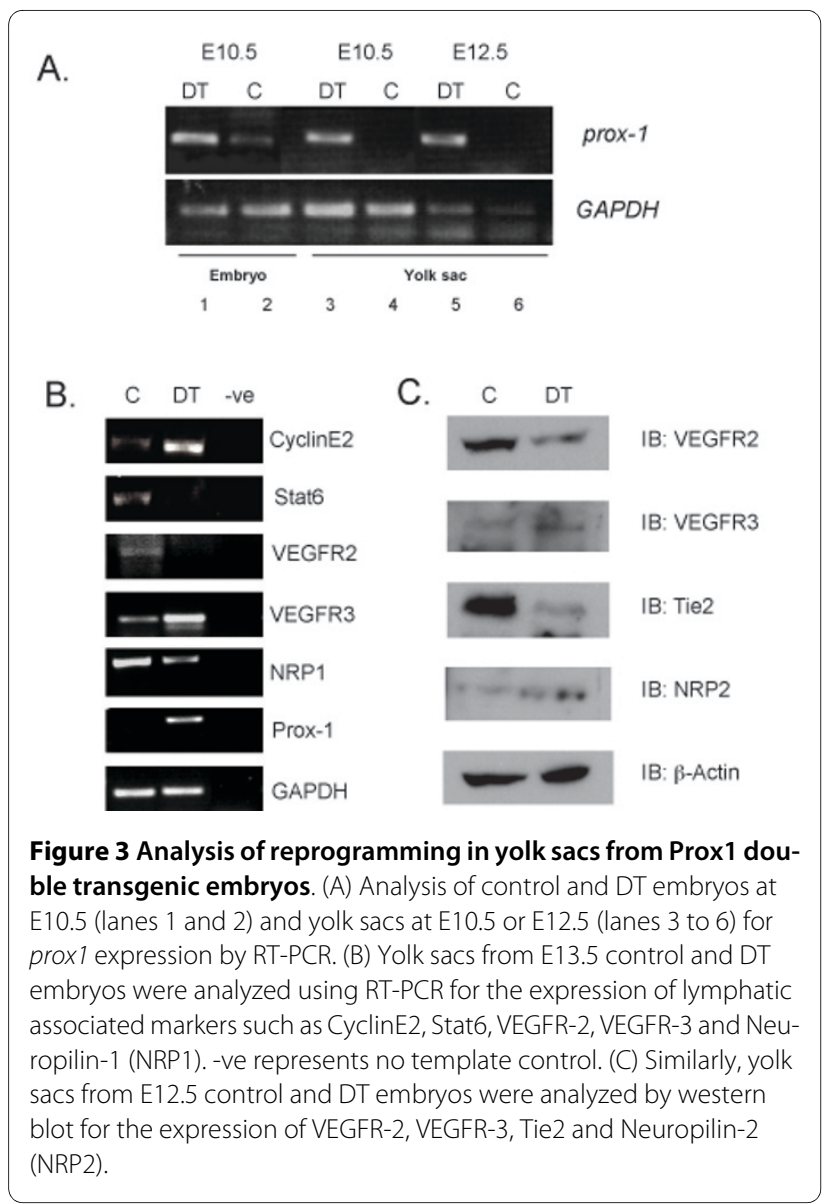

occurs. In our model, one can hypothesize that the overexpression of Prox 1 may influence junctional proteins involved in maintaining the integrity of cell-cell contacts; alterations in these same complexes may result in changes in vascular permeability leading to a more permeable, lymphatic-like state [15]. Indeed, upon Prox1 overepression changes in junctional proteins are observed away from the normal molecular profile associated with control embryos. Previous studies have shown that increases as well as decreases in junction protein levels have been shown to be associated with an increase in permeability $[16,17]$, suggesting that a balance is required in order to maintain the integrity of the cell-cell interface. Recent data has found that the overexpression of Prox 1 in colon epithelial cells result in dysplasia and transformation that correlates with changes in cellular adhesion [11]. Furthermore, conditional deletion of Prox1 results in aberrant junctional complex formation and abnormal pericyte association [14]. These lines of evidence point to the importance of cell-cell integrity in the regulation of vascular permeability. Moreover, the controlled expression of Prox1 is critical for normal lymphatic development in the embryo.

It is clear that the measured expression of Prox 1 as well as other lymphangiogenic factors during embryonic development is essential for the proper formation of the lymphatic vasculature. Not surprisingly, compromising this regulated expression has been found to result in negative clinical consequences. For example, the growth of lymphatic endothelium in tumors is a result of the presence of VEGF-C, which acts on lymph vessels to enhance metastasis [18]. Other events include the transformation and cellular reprogramming of spindle-like lesional cells by Karposi sarcoma herpesvirus (KHSV)/human herpesvirus-8 (HHV8). Interestingly, gene profiling of KHSV infected lesional cells produce a signature similar to that derived from a lymphatic endothelial cell; targets that include Prox1, LYVE-1 and VEGFR-3. This suggests that Karposi sarcoma genetically mimics an expression pattern similar to a differentiated lymphatic endothelial cell [19-21].

\section{Conclusions}

In conclusion, we extend the previous in vitro studies that characterized the molecular profile of reprogrammed vascular endothelial cells that overexpress the Prox 1 transcription factor. Furthermore, we also demonstrate that the tight regulation of Prox1 is critical for normal embryonic lymphatic development. While the expression pattern of Prox1 in our model does not fully recapitulate the GFP expression found in the Tie1-GFP mouse model, it is clear that when Prox1 is overexpressed in our tissue specific model it results in aberrant lymph sac development, hemorrhaging, edema, anemia and ultimately, embryonic lethality. In a corresponding study, Johnson et al. demonstrated that Prox1 was essential for the maintenance of the lymphatic phenotype, where a global temporal deletion of Prox1 during mid-development effectively deprograms lymphatic structures to that of a vascular endothelial-like phenotype, assessed by surface markers such as Podoplanin and SLC [14]. Our data presented here complement the work by Johnston et al., whereby the tissue specific overexpression of Prox1 reprograms similar markers to a more lymphatic-like profile. Furthermore, we elucidate some of the molecular components that accompany vascular endothelial cell reprogramming involved in early lymphatic differentiation and development. The development of our unique model complements and extends the current viewpoint of Prox1 being an important initiator of lymphatic development via its ability to reprogram endothelial cells to a specific molecular profile. 


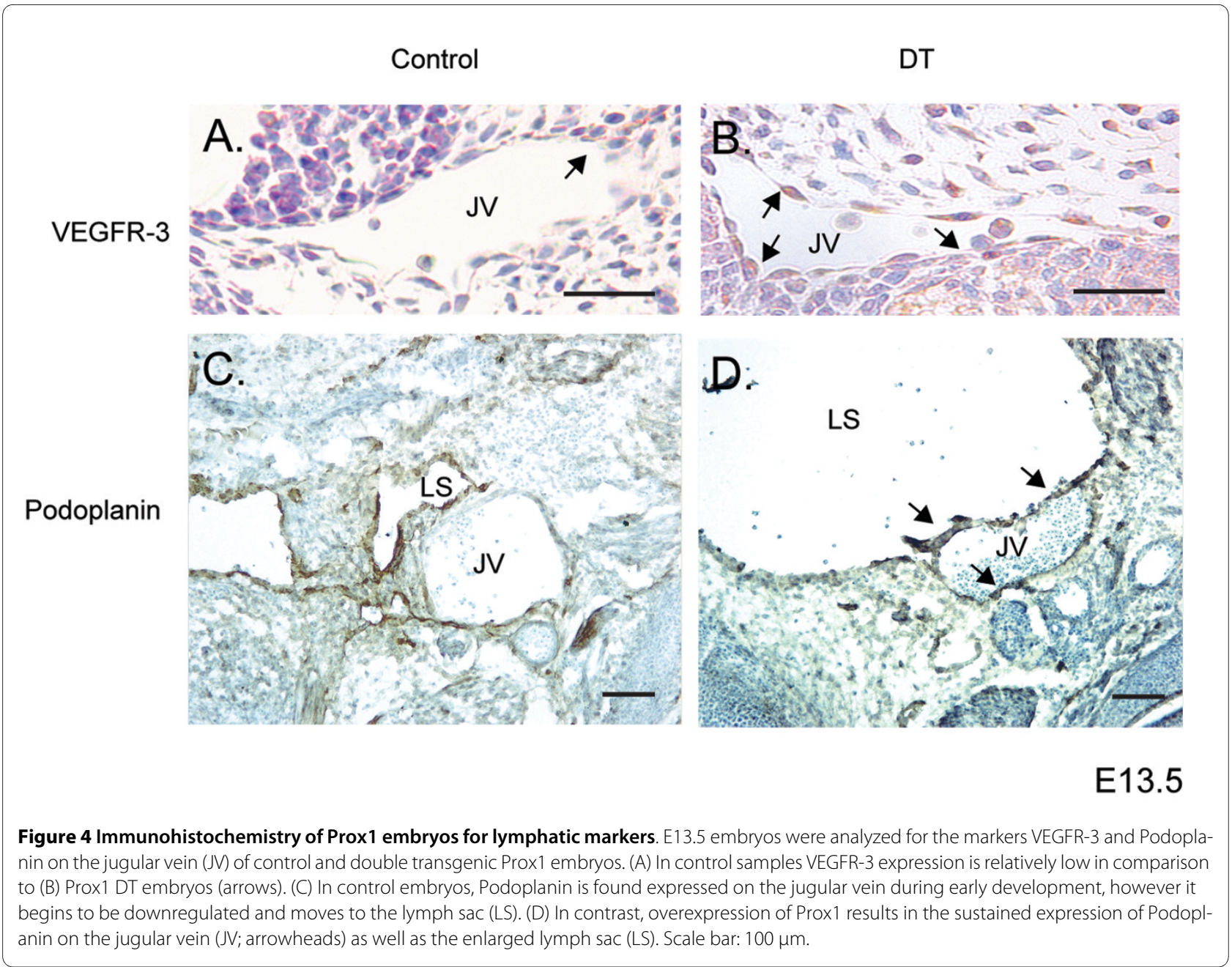

\section{Methods}

\section{Generation of mice}

The Animal Care and Ethics Committee approved all animals and protocols that were used. The construction of the tie1 and tie 2 tTA driver transgene has been previously described [22]. The prox1 cDNA was inserted into the $p T e{ }^{O S}$ responder construct and transgenic animals were derived at the McGill Transgenic Facility, Montreal, Quebec, Canada. Driver and responder transgenic animals were bred to generate bigenic embryos. Embryos were genotyped and wild type, single and double transgenics in the presence of doxycycline was used as controls relative to double transgenics without doxycycline. Doxycycline treatment involved the addition of $100 \mu \mathrm{g} / \mathrm{mL}$ of doxycycline $/ 5 \%$ sucrose in the drinking water and changed at least twice per week.

\section{Immunofluorescence and immunohistochemistry}

Embryos were prepared by fixing in $4 \%$ paraformaldyhyde, followed by incubation in 30\% sucrose and mounted in OCT for cryosectioning. Sections were treated with $0.5 \%$ TritonX-100/PBS and blocked in $5 \% \mathrm{BSA} / 10 \%$ goat serum prior to antibody incubation. Antibodies used were anti-Prox1 (102PA30, RDI), Podoplanin (clone 8.1.1) and VEGFR-3 (16-5988, eBiosciences). Immunohistochemistry was counterstained with Harris hematoxylin.

\section{RT-PCR analysis}

Yolk sacs were placed in Trizol (GibcoBRL) and processed following manufacturers protocol. In brief, tissues were homogenized and $200 \mu \mathrm{L}$ of chloroform was added per $1 \mathrm{~mL}$ Trizol. Following centrifugation at 10,000 $\times \mathrm{g}$ for 15 minutes at $4^{\circ} \mathrm{C}$, the upper phase was removed and $300 \mu \mathrm{L}$ of $100 \%$ ethanol was added per $1 \mathrm{~mL}$ of Trizol. After 5 minutes incubation at room temperature, RNA was isolated by centrifugation at 2,000 $\times \mathrm{g}$ for 5 minutes at $4^{\circ} \mathrm{C}$. Proteins were then precipitated from the phenolethanol supernatant by $1.5 \mathrm{~mL}$ isopropyl alcohol per $1 \mathrm{~mL}$ Trizol. After 10 minutes incubation at room temperature, protein precipitate was isolated at $12,000 \times \mathrm{g}$ for $10 \mathrm{~min}$ utes at $4^{\circ} \mathrm{C}$. Reverse transcriptase was performed as per 

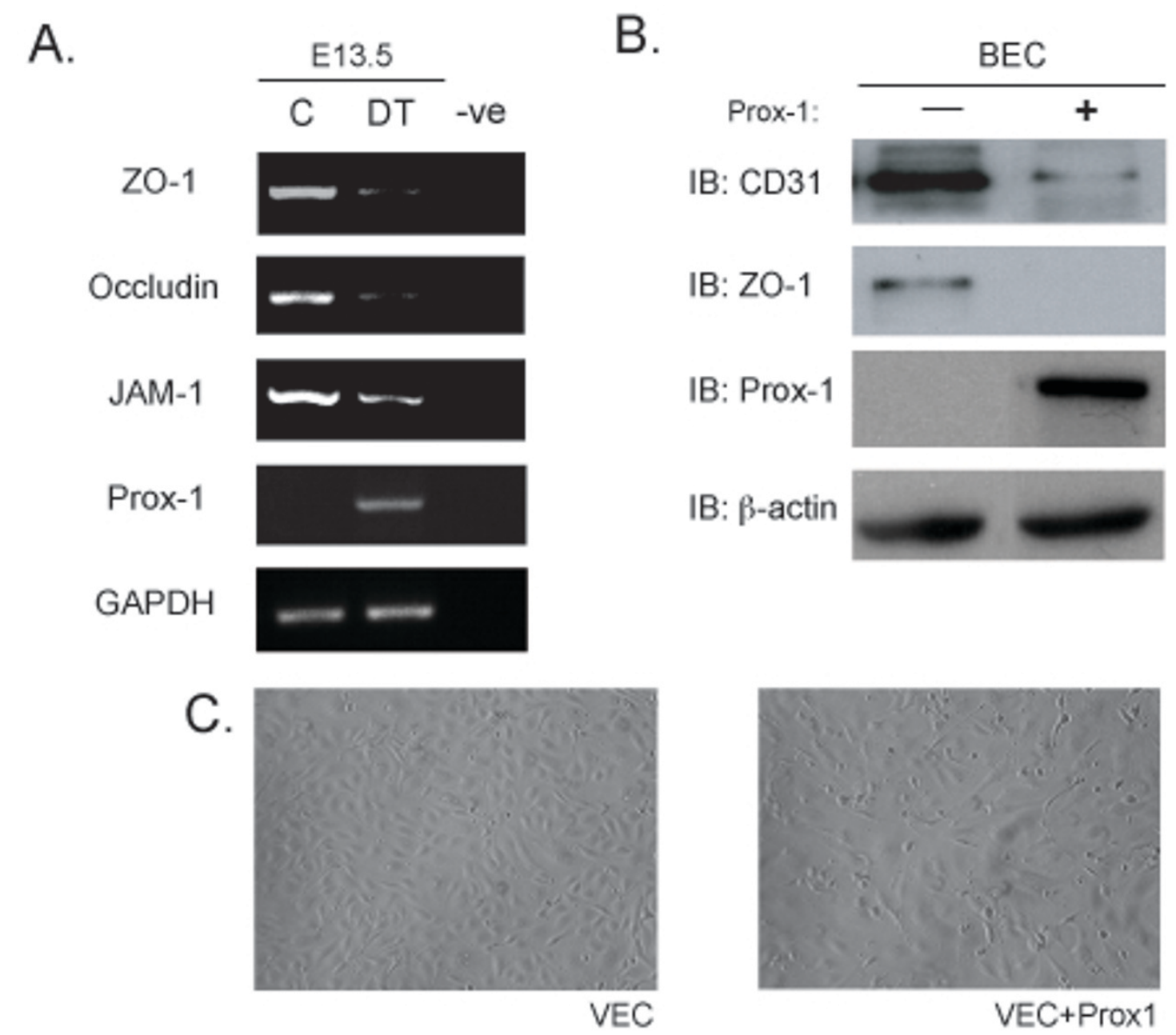

IB: ZO-1

IB: Prox-1
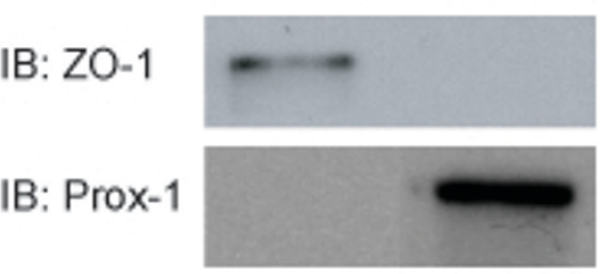

IB: $\beta$-actin
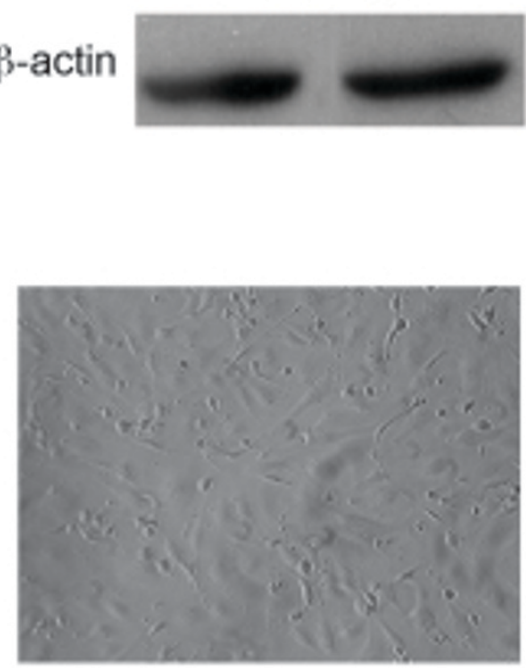

VEC+Prox1

D.

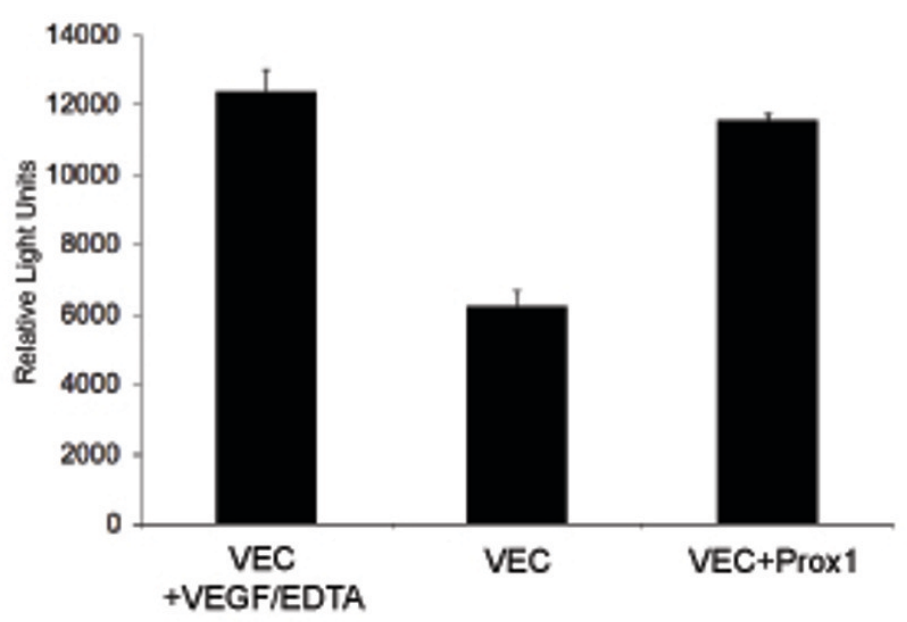

Figure 5 Overexpression of Prox 1 results in changes in junctional proteins in vivo and in vitro. (A) RT-PCR analysis was performed on yolk sacs derived from E13.5 embryos to targets such as ZO-1, Occludin and JAM-1. (B) Similarly, in vitro analysis shows that overexpresion of Prox1 in vascular endothelial cells decreases junctional protein levels. (C) Bright field microscopy of VECs transfected with Prox1 (10x mag) (D) Vascular endothelial cells that overexpress Prox 1 display an increase in permeability measured by FITC-albumin transit in a Boyden chamber assay. Figure is representative of three experiments with all samples run in triplicate. Error bars represent standard deviation of the mean. $P<0.05$. 
manufacturers protocol (Qiagen). Negative control represents no template. PCR primers used were as follows:

mVEGFR-2 For: ATGAAATTGAGCTATCTGCC, Rev: CCACTGGATGTGGTGCAGGG

mNeuropilin-1For: GCAATAGCAAAAGAAGGTTT,

Rev: ACCATGCCCAACAATCCAGA

mStat6 For: ATCCAGCTTCAGGCCCTGTC, Rev:

TCTATCTGTGAGGAGCCATC

mCyclinE2 For: ATCCAGTCTACAGATTCCGA, Rev:

ATCCAGTCTACAGATTCCGA

Prox1 For: ATGCCTGACCATGACAGC, Rev:

GGGAAGCTTTTGCTTGCG

mJAM-1 For: CCGAGTGGAGTGGAAGTTCGTCC,

Rev: AGGAACGACGAGGTCTGTTTGAATTC

mOccludin For: ACTTCAGGCAGCCTCGGTACAG,

Rev: CTCCCGCAACTGGCATCTCTCTAA

mZO-1For: TCACAGGGCTCCTGGGTTTGGAT,

Rev: CTAGTGACTGAATTTCTGAAATGTCATCT

GAPDH For: CTGCACCACCAACTGCTTAG, Rev: TCTCATCATACTTGGCAGGT

\section{Western analysis}

Venous endothelial cells used in this study have been previously characterized [10]. Proteins were lysed in RIPA buffer for 30 minutes on ice $\left(10 \mathrm{mM} \mathrm{NaH}_{2} \mathrm{PO}_{4} \mathrm{pH} 7.5,150\right.$ $\mathrm{mM} \mathrm{NaCl}, 1 \%$ NP-40, 0.1\% SDS, $1 \%$ Sodium Deoxycholate, $10 \mathrm{mM} \mathrm{NaF}, 2 \mathrm{mM}$ EDTA, Protease Inhibitor cocktail (Complete-EDTA free, Roche USA), and $10 \mathrm{mM}$ sodium orthovanadate, cleared by centrifugation and the supernatants collected for further analysis. Equal amounts of lysates were resuspended with 2xSDS loading buffer and separated via SDS-PAGE. Proteins were transferred to PVDF, blocked with 3\% milk/tris buffered saline, incubated with the appropriate primary and secondary antibody conjugated to horse radish peroxidase, and developed via enhanced chemiluminescence (Pierce). Antibodies used include Prox-1 (07-537, Upstate), CD31 (557355, BD Biosciences), ZO-1 (40-2300, Zymed) and $\beta$ actin (AC15, Sigma).

\section{Permeability assay}

This protocol is a modification of the permeability assay by Chemicon (ECM640). To generate Prox1 overexpressing cells, bovine VECs [10] were transfected with a human Prox1 cDNA via Lipofectamine 2000 as per manufacturers protocol (Gibco). $2 \times 10^{5}$ cells were plated in a $3 \mu \mathrm{m}$ pore size transwell with no substrate. Positive controls included $100 \mathrm{ng} / \mathrm{mL}$ of VEGF-A/25 mM EDTA in the top well. Also included in the top well was $250 \mu \mathrm{g} / \mathrm{mL}$ of FITC-Albumin to quantitate permeability through the cell monolayer. After the cells were incubated for 48 hours, passage of FITC-Albumin $(250 \mu \mathrm{g} / \mathrm{mL})$ into the bottom chamber was detected at an excitation/emission ratio of 488/530. $n=3$, performed in triplicate.

\section{Statistical analysis}

Data was presented as standard errors from the mean. Statistical significance was taken at $P<0.05$ using an unpaired, two-tailed Student's $t$-test.

\section{Additional material}

\begin{abstract}
Additional file 1 Prox 1 overexpression in control and bigenic
embryos. ( $A$ and $B$ ) Control and ( $C$ and $D$ ) bigenic embryos at $E 10.5$ were analyzed for Prox-1 expression (red) and smooth muscle actin (SMA, green). Of note, at this stage in development SMA is found to associate with the dorsal aorta (Panel C, arrow) but not the cardinal vein, thereby providing a simple landmark for identification. Significantly, (A) control embryos only display Prox-1 expression from the cardinal vein, however (C) DT embryos are positive for Prox-1 expression both the dorsal aorta (arrow) and the cardinal vein. (B and D) Sections have been counterstained with DAPI. CV: cardinal vein, DA: dorsal aorta, D: dorsal, L: lateral. Scale bar: A and B $100 \mu \mathrm{m}, \mathrm{C}$ and D $200 \mu \mathrm{m}$
\end{abstract}

\section{Authors' contributions}

HK was responsible for the experimental design, execution, and writing of this manuscript; VPKHN provided cell lines; TP constructed the transgenic construct; MC provided animal husbandry and technical support; KA Collaborator; DJD principle investigator. All authors read and approved the final manuscript.

\section{Acknowledgements}

H.K. was supported by a postdoctoral fellowship from the Susan G. Komen Foundation (PDF0600259); V.P.K.H.N is supported by a Canadian Institutes of Health Research, Banting and Best Canadian Graduate Scholar Award; D.J.D. is the Canadian Research Chair for Angiogenic and Lymphangiogenic Signaling Funding was provided by the Heart and Stroke Foundation of Canada (T-6123). Many thanks to Drs. Paul Van Slyke, Annie Bourdeau and Jennifer Alami for critical reading and helpful input.

\section{Author Details}

1Sunnybrook Research Institute University of Toronto 2075 Bayview Avenue Toronto, Ontario, M4N 3M5, Canada, ${ }^{2 B i o m e d i c u m ~ H e l s i n k i ~ H a a r t m a n ~ I n s t i t u t e ~}$ PO Box 63 (Haartmaninkatu 8) Fl-00014 University of Helsinki, Helsinki, Finland and ${ }^{3}$ Division of Experimental Oncology CePO, CHUV and University of Lausanne 155, Chemin des Boveresses CH-1066 Epalinges Switzerland

Received: 19 August 2009 Accepted: 28 June 2010

Published: 28 June 2010

\section{References}

1. Aitsebaomo J, Portbury AL, Schisler JC, Patterson C: Brothers and sisters: molecular insights into arterial-venous heterogeneity. Circ Res 2008, 103:929-39.

2. Wigle JT, Harvey N, Detmar M, Lagutina I, Grosveld G, Gunn MD, Jackson DG, Oliver G: An essential role for Prox1 in the induction of the lymphatic endothelial cell phenotype. Embo J 2002, 21:1505-13.

3. Wigle JT, Oliver G: Prox 1 function is required for the development of the murine lymphatic system. Cell 1999, 98:769-78.

4. Prevo R, Banerji S, Ferguson DJ, Clasper S, Jackson DG: Mouse LYVE-1 is an endocytic receptor for hyaluronan in lymphatic endothelium. J Biol Chem 2001, 276:19420-30.

5. Schacht V, Ramirez MI, Hong YK, Hirakawa S, Feng D, Harvey N, Williams M, Dvorak AM, Dvorak HF, Oliver G, et al:: T1alpha/podoplanin deficiency disrupts normal lymphatic vasculature formation and causes lymphedema. Embo J 2003, 22:3546-56.

6. Kaipainen A, Korhonen J, Mustonen T, van Hinsbergh VW, Fang GH, Dumont D, Breitman M, Alitalo K: Expression of the fms-like tyrosine kinase 4 gene becomes restricted to lymphatic endothelium during development. Proc Natl Acad Sci USA 1995, 92:3566-70.

7. Petrova TV, Makinen T, Makela TP, Saarela J, Virtanen I, Ferrell RE, Finegold DN, Kerjaschki D, Yla-Herttuala S, Alitalo K: Lymphatic endothelial reprogramming of vascular endothelial cells by the Prox-1 homeobox transcription factor. Embo J 2002, 21:4593-9. 
8. Hong YK, Harvey N, Noh YH, Schacht V, Hirakawa S, Detmar M, Oliver G: Prox 1 is a master control gene in the program specifying lymphatic endothelial cell fate. Dev Dyn 2002, 225:351-7.

9. Harvey NL, Srinivasan RS, Dillard ME, Johnson NC, Witte MH, Boyd K, Sleeman MW, Oliver G: Lymphatic vascular defects promoted by Prox 1 haploinsufficiency cause adult-onset obesity. Nat Genet 2005, 37:1072-81.

10. Nguyen VP, Chen SH, Trinh J, Kim H, Coomber BL, Dumont DJ: Differential response of lymphatic, venous and arterial endothelial cells to angiopoietin-1 and angiopoietin-2. BMC Cell Biol 2007, 8:10.

11. Petrova TV, Nykanen A, Norrmen C, Ivanov Kl, Andersson LC, Haglund C, Puolakkainen P, Wempe F, von Melchner H, Gradwohl G, et al: Transcription factor PROX1 induces colon cancer progression by promoting the transition from benign to highly dysplastic phenotype. Cancer Cell 2008, 13:407-19.

12. YXu, Yuan L, Mak J, Pardanaud L, Caunt M, Kasman I, Larrivee B, Del Toro R, Suchting S, Medvinsky A, et al:: Neuropilin-2 mediates VEGF-C-induced lymphatic sprouting together with VEGFR3. J Cell Bio/ 188:115-30.

13. Francois M, Caprini A, Hosking B, Orsenigo F, Wilhelm D, Browne C, Paavonen K, Karnezis T, Shayan R, Downes M, et al:: Sox18 induces development of the lymphatic vasculature in mice. Nature 2008, 456:643-7.

14. Johnson NC, Dillard ME, Baluk P, McDonald DM, Harvey NL, Frase SL, Oliver G: Lymphatic endothelial cell identity is reversible and its maintenance requires Prox1 activity. Genes Dev 2008, 22:3282-91.

15. Murfee WL, Rappleye JW, Ceballos M, Schmid-Schonbein GW: Discontinuous expression of endothelial cell adhesion molecules along initial lymphatic vessels in mesentery: the primary valve structure. Lymphat Res Biol 2007, 5:81-9.

16. Abe T, Sugano E, Saigo Y, Tamai M: Interleukin-1 beta and barrier function of retinal pigment epithelial cells (ARPE-19): aberrant expression of junctional complex molecules. Invest Ophthalmol Vis Sci 2003, 44:4097-104.

17. Laukoetter MG, Nava P, Lee WY, Severson EA, Capaldo CT, Babbin BA, Williams IR, Koval M, Peatman E, Campbell JA, et al.: JAM-A regulates permeability and inflammation in the intestine in vivo. JExp Med 2007, 204:3067-76.

18. Tammela T, Alitalo K: Lymphangiogenesis: Molecular mechanisms and future promise. Cell 2010, 140:460-76.

19. Jussila L, Valtola R, Partanen TA, Salven P, Heikkila P, Matikainen MT, Renkonen R, Kaipainen A, Detmar M, Tschachler E, et al:: Lymphatic endothelium and Kaposi's sarcoma spindle cells detected by antibodies against the vascular endothelial growth factor receptor-3. Cancer Res 1998, 58:1599-604.

20. Wang HW, Trotter MW, Lagos D, Bourboulia D, Henderson S, Makinen T, Elliman S, Flanagan AM, Alitalo K, Boshoff C: Kaposi sarcoma herpesvirusinduced cellular reprogramming contributes to the lymphatic endothelial gene expression in Kaposi sarcoma. Nat Genet 2004, 36:687-93.

21. Weninger W, Partanen TA, Breiteneder-Geleff S, Mayer C, Kowalski H, Mildner M, Pammer J, Sturzl M, Kerjaschki D, Alitalo K, et al.: Expression of vascular endothelial growth factor receptor-3 and podoplanin suggests a lymphatic endothelial cell origin of Kaposi's sarcoma tumor cells. Lab Invest 1999, 79:243-51.

22. Sarao R, Dumont DJ: Conditional transgene expression in endothelial cells. Transgenic Res 1998, 7:421-7.

doi: 10.1186/1471-213X-10-72

Cite this article as: Kim et al., Embryonic vascular endothelial cells are malleable to reprogramming via Prox 1 to a lymphatic gene signature BMC Developmental Biology 2010, 10:72

\section{Submit your next manuscript to BioMed Central and take full advantage of:}

- Convenient online submission

- Thorough peer review

- No space constraints or color figure charges

- Immediate publication on acceptance

- Inclusion in PubMed, CAS, Scopus and Google Scholar

- Research which is freely available for redistribution

Submit your manuscript at www.biomedcentral.com/submit
C Biomed Central 